\title{
Crystallographic and Nanomechanical Analysis by Correlative In-situ AFM \& SEM
}

P. Frank ${ }^{1}$, M. Leitner ${ }^{1}$, S. Hummel ${ }^{1}$, N. Hosseini ${ }^{2}$, Y. Wang ${ }^{3}$, R. Winkler ${ }^{4}$, G. E. Fantner ${ }^{2}$, H. Plank ${ }^{4}$, Y. Zeng $^{3}$ and C. H. Schwalb ${ }^{1}$

1. GETec Microscopy GmbH, Vienna, Austria

2. Laboratory for Bio- and Nano-Instrumentation, Institute for Bioengineering, EPFL, Switzerland

3. The State Key Lab of High Performance Ceramics and Superfine Microstructure, SICCAS, China

4. Institute for Electron Microscopy and Nanoanalysis, Graz University of Technology, Austria.

Combining different microscopic and spectroscopic analysis techniques has been a field with much activity over the past decade. The integration of different methods into one instrument allows simultaneous access to complementary information about the sample helping to yield a more complete picture. Especially highly localized probing of mechanical, electrical, chemical and crystallographic properties on the nanoscale represents a key success factor for gaining new insights in the micro and nano world.

This work focuses on the combination of crystallographic analysis by means of electron backscatter diffraction (EBSD) on focused ion beam (FIB) polished surfaces and correlative sub-nm topography analysis in the field of zirconia-based ceramics. Zirconia $\left(\mathrm{ZrO}_{2}\right)$-based ceramics are strong, hard and inert and have low thermal conductivity and good biocompatibility. Due to these properties they have found wide applicability in the fields of optical communication, thermal barrier coatings, automobile industry and femoral implants. However, phase transformation processes in zirconia-based ceramics which can either strengthen or weaken the material are barely understood due to the lack of correlative crystallographic phase and morphology information.

A unique atomic force microscope (AFM) -the AFSEM ${ }^{\text {TM}}$ - designed for seamless integration into scanning electron microscopes (SEM) or dual beam systems is used for the correlative in-situ AFM/SEM/EBSD analysis at the exact same sample position without the need of air exposure or sample transfer (Figure 1). Its compact design and the use of self-sensing cantilevers with electrical readout [1] allows for simultaneous operation of SEM, FIB and AFM inside the vacuum chamber. For the first time, the martensitic transition in zirconia coatings was investigated by combination of in-situ electron backscatter diffraction and in-situ atomic force microscopy [2]. In addition to the EBSD mapping, which shows the local occurrence of phase transition, in-situ AFM can now be utilized to analyze phasetransformation-induced changes in topography with sub-nm resolution. The direction of residual stress was revealed by the periodic corrugation patterns of AFM images, matched precisely with the $10^{\circ}$ deflection of the basal texture (Figure 2). This finding was further confirmed by an in-situ reverse transformation from the monoclinic phase to tetragonal phase and the formation of vertical microcracks after stress release during heat treatment.

We also demonstrate, that in-situ correlative analysis with AFSEM ${ }^{\mathrm{TM}}$ in an SEM can be extended into the third dimension to measure nanomechanical properties of soft materials. To achieve this, FIB slicing and mapping of nanomechanical properties using the AFSEM ${ }^{\mathrm{TM}}$ is performed in repetitive steps to build up a 3-dimensional elasticity map. These applications illustrate the suitability and capability of $\mathrm{AFSEM}^{\mathrm{TM}}$ as a characterization tool for nanomechanical analysis. 


\section{References:}

[1] M Dukic, JD Adams and GE Fantner, Scientific Reports 5 (2015) 16393

[2] Y Wang et al, submitted to Scientific Reports (2018)

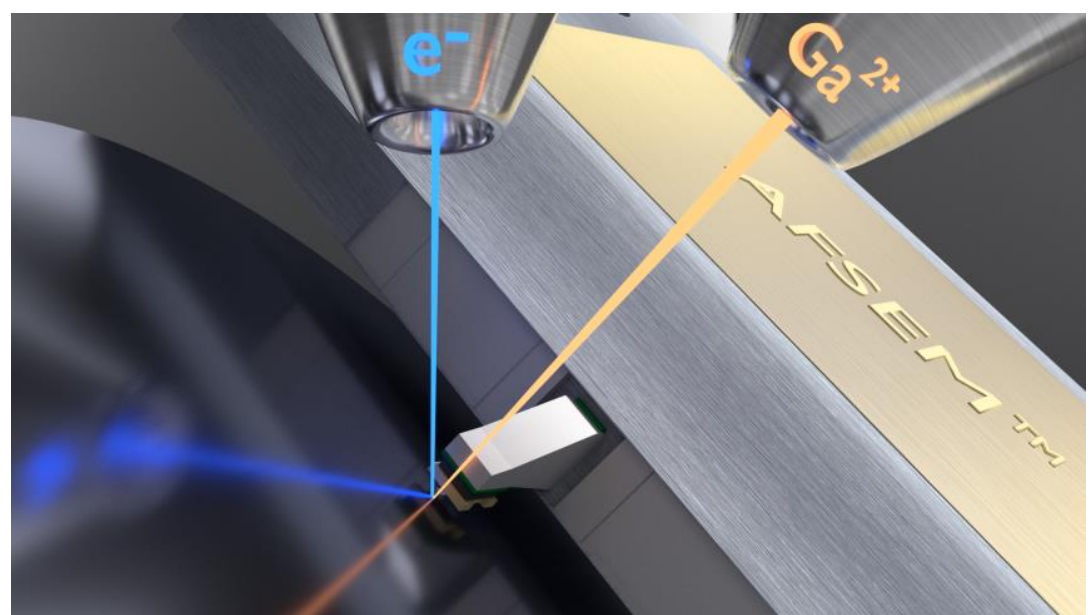

Figure 1. Schematics of correlative analysis in a dual beam system using SEM, FIB, and AFSEM ${ }^{\mathrm{TM}}$ in an interactive experiment

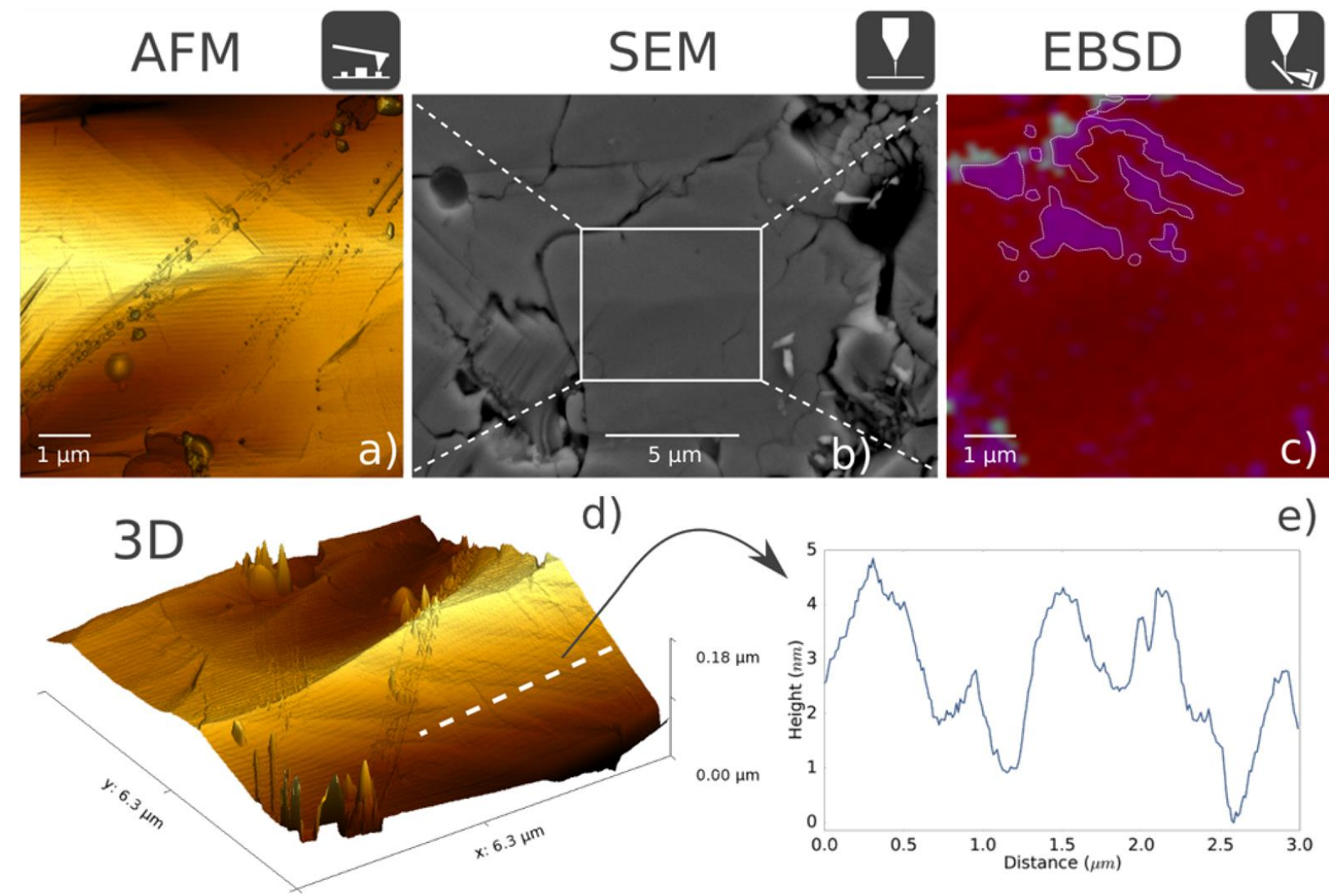

Figure 2. AFM image (a), correlative SEM image (b) and EBSD crystallographic analysis of $\mathrm{ZrO}_{2}$ sample in phase transformed. (d) 3D topography showing phase transformation-induced changes of topography up to $5 \mathrm{~nm}$ as shown by the (e) line cross section in transformed areas. 